\title{
Pheromone binding by polymorphic mouse major urinary proteins
}

\author{
SCOTT D. SHARROW, JEFFREY L. VAUGHN, LUKÁŠ ŽÍDEK ${ }^{1}$, MILOS V. NOVOTNY, \\ AND MARTIN J. STONE \\ Institute for Pheromone Research and Department of Chemistry, Indiana University, \\ Bloomington, Indiana 47405-0001
}

(Received February 5, 2002; Final Revision May 28, 2002; Accepted June 24, 2002)

\begin{abstract}
Mouse major urinary proteins (MUPs) have been proposed to play a role in regulating the release and capture of pheromones. Here, we report affinity measurements of five recombinant urinary MUP isoforms (MUPs-I, II, VII, VIII, and IX) and one recombinant nasal isoform (MUP-IV) for each of three pheromonal ligands, ( \pm )-2-sec-butyl-4,5-dihydrothiazole (SBT), 6-hydroxy-6-methyl-3-heptanone (HMH), and ( \pm )dehydro-exo-brevicomin (DHB). Dissociation constants for all MUP-pheromone pairs were determined by isothermal titration calorimetry, and data for SBT were corroborated by measurements of intrinsic protein fluorescence. We also report the isolation of MUP-IV protein from mouse nasal extracts, in which MUP-IV mRNA has been observed previously. The affinity of each MUP isoform for SBT $\left(\mathrm{K}_{\mathrm{d}} \sim 0.04\right.$ to $\left.0.9 \mu \mathrm{M}\right)$ is higher than that for DHB $\left(\mathrm{K}_{\mathrm{d}} \sim 26\right.$ to $\left.58 \mu \mathrm{M}\right)$, which in turn is higher than that for HMH $\left(\mathrm{K}_{\mathrm{d}} \sim 50\right.$ to 200 $\mu \mathrm{M})$. Isoforms I, II, VIII, and IX show very similar affinities for each of the ligands. MUP-VII has approximately twofold higher affinity for SBT but approximately twofold lower affinity for the other pheromones, whereas MUP-IV has $\sim 23$-fold higher affinity for SBT and approximately fourfold lower affinity for the other pheromones. The variations in ligand affinities of the MUP isoforms are consistent with structural differences in the binding cavities of the isoforms. The data indicate that the concentrations of available pheromones in urine may be influenced by changes in the expression levels of urinary MUPs or the excretion levels of other MUP ligands. The variation in pheromone affinities of the urinary MUP isoforms provides only limited support for the proposal that MUP heterogeneity plays a role in regulating profiles of available pheromones. However, the binding data support the proposed role of nasal MUPs in sequestering pheromones and possibly transporting them to their receptors.
\end{abstract}

Keywords: Binding affinity; isothermal titration calorimetry; major urinary proteins; pheromones; selectivity

Supplemental material: See www.proteinscience.org.

Among different mammalian species, the role of chemical communication has been best documented in rodents. In the house mouse, Mus domesticus, the known pheromonal ef-

Reprint requests to: Martin J. Stone, Department of Chemistry, Indiana University, Bloomington, Indiana 47405-0001, USA; e-mail: mastone@ indiana.edu; fax: (812) 855-8300 or Milos V. Novotny, Department of Chemistry, Indiana University, Bloomington, Indiana 47405-0001, USA; e-mail: novotny@indiana.edu; fax: (812) 855-8300.

${ }^{1}$ Present address: The National Centre for Biomolecular Research, Faculty of Science, Masaryk University, Kotlarska 2, 61137 Brno, Czech Republic.

Article and publication are at http://www.proteinscience.org/cgi/doi/ 10.1110/ps.0204202. fects range from a temporary behavioral modification, such as intermale aggression (Novotny et al. 1985) and dominance signaling (Novotny et al. 1990a), to a longer-lasting physiological change represented by estrus synchrony (Jemiolo et al. 1986), puberty acceleration (Novotny et al. 1999a,b), or puberty delay (Novotny et al. 1986; Jemiolo and Novotny, 1994). The murine chemical communication system relies on various pheromones (usually small, relatively hydrophobic molecules) that primarily originate from the urine or glandular secretions of a donor animal (Novotny et al. 1990b). The primary sites of pheromone action are the membrane-bound $\mathrm{G}$ protein-coupled receptors located in 
the neurons of the olfactory mucosa and the vomeronasal organ (Buck and Axel 1991; Dulac and Axel 1995). However, a group of small, soluble proteins belonging to the lipocalin family seems to assist the pheromone communication system. These so-called major urinary proteins (MUPs; Finlayson and Baumann 1958; Sampsell and Held 1985) are expressed at the sites of pheromone excretion (liver and kidney), whereas some MUP mRNA is also present in the nasal mucosa and lachrymal, parotid, sublingual, and submaxillary glands (Shahan et al. 1987). MUPs bind to pheromonal ligands (Bacchini et al. 1992; Robertson et al. 1993). The typical concentration of MUPs in mouse urine is 1 to $5 \mathrm{mg} / \mathrm{mL}$, indicating a substantial metabolic cost of MUP expression (Ferrari et al. 1997). Laboratory mice (inbred strains) can express a number of different MUP isoforms, which are encoded by different genes (with $>85 \%$ sequence identity; Shahan et al. 1987). The profile of expressed isoforms may vary substantially, depending on strain, gender, and hormonal levels (Clissold and Bishop 1982; Johnson et al. 1995; Robertson et al. 1996). A markedly enhanced MUP polymorphism has recently been observed in wild mouse populations (Payne et al. 2001; Hurst et al. 2001a). As noted by Hurst et al. (1998) and Beynon et al. (1999) in a series of ligand displacement studies, MUPs play a role in slow release of pheromones. At the site of pheromone perception, MUPs or other lipocalins may assist in capturing pheromones in the nasal cavity, preconcentration and/or transport functions, or other "perireceptor functions" (Pelosi 1994).

The functional roles of the many MUP isoforms are currently unclear. One hypothesis is that the isoforms may differentially bind and release different pheromones (Marie et al. 2000; Timm et al. 2001) so that, by regulating MUP expression levels, the animals could indirectly regulate the levels of available pheromones. This hypothesis requires that the MUP isoforms have different affinities and/or specificities for the pheromones. Currently, only sparse MUPpheromone binding data have been reported because of the difficulty in isolating pure isoforms and pheromones from natural sources. However, a recent report (Marie et. al. 2000) showed that MUP isoforms with a single-amino-acid difference had differential affinities for an unnatural fluorescent ligand. This result indicated that MUP isoforms might have affinity differences for natural pheromones.

Here, we present data showing significant variations in both affinity and specificity of six different MUP isoforms binding to each of the three natural, male-derived pheromones, 2-sec-butyl-4,5-dihydrothiazole (SBT), 6-hydroxy6-methyl-3-heptanone (HMH), and dehydro-exo-brevicomin (DHB). SBT and DHB have parallel biological activities in inducing estrus synchrony (Jemiolo et al. 1986), accelerating puberty in females (Novotny et al. 1999b), and causing inter-male aggression (Novotny et al. 1985). HMH has an accelerating effect on female puberty onset (Novotny et al. 1999a). Among the six MUP isoforms tested, isoforms I, II, VII, VIII, and IX represent forms most abundant in the urine of laboratory mice, whereas MUP-IV is expressed in the nasal mucus. MUP-IV shows a distinct difference in binding specificity from the urinary forms. The implications of the binding data for the function of MUPs in pheromonal transport are discussed.

\section{Results}

Previous studies of MUP-pheromone interactions have been limited by the availability of pure MUP isoforms and pure pheromones. To circumvent these problems, we have expressed each of the six MUP isoforms in Escherichia coli and have synthesized each of the three pheromones. The expression of MUP-I in E. coli has been described previously (Zidek et al. 1999). For the current study, the other isoforms were expressed and purified using a similar protocol. In all cases, the observed molecular masses and isoelectric points are consistent with the predicted values (Table 1).

The three pheromones, SBT, HMH, and DHB (Fig. 1), were obtained by chemical synthesis. The SBT used here is a racemic mixture. SBT is isolated from urine already as a racemic mixture (Novotny et al. 1995), and the two enantiomers have been shown to bind to MUP-I with similar affinities (Zidek et al. 1999). Although it is possible to synthesize optically active SBT, it racemizes readily, precluding binding or activity studies with the pure enantiomers (Novotny et al. 1995). The DHB used here is a racemic mixture of the $(\mathrm{R}, \mathrm{R})$ and $(\mathrm{S}, \mathrm{S})$ stereoisomers. DHB is isolated from urine as its $(\mathrm{R}, \mathrm{R})$-form. Although only the $(\mathrm{R}, \mathrm{R})$ isomer displays pheromonal activity (Novotny et al. 1995), both the $(\mathrm{R}, \mathrm{R})$ and $(\mathrm{S}, \mathrm{S})$ stereoisomers bind to MUP-I (Bacchini et al. 1992). HMH is nonchiral but exists in equilibrium between the open (hydroxyketone) and closed (hydroxyfuran) tautomeric forms (Fig. 1; Novotny et al. 1999b).

Table 1. Molecular mass and isoelectric point data for recombinant MUP isoforms

\begin{tabular}{lcccc}
\hline Isoform & $\mathrm{MW}^{\mathrm{a}}$ & $\mathrm{MW}^{\mathrm{b}}$ & $\mathrm{pI}^{\mathrm{a}}$ & $\mathrm{pI}^{\mathrm{b}}$ \\
\hline MUP-I & $18,693 \pm 3$ & $18,691.8$ & 4.7 & 4.7 \\
MUP-II & $18,705 \pm 4$ & $18,706.8$ & 4.7 & 4.7 \\
MUP-IV & $18,817 \pm 1$ & $18,817.0$ & 5.3 & 5.5 \\
MUP-VII & $18,643 \pm 3$ & $18,646.0$ & 4.7 & 4.7 \\
MUP-VIII & $18,692 \pm 5$ & $18,695.0$ & 4.6 & 4.6 \\
MUP-IX & $18,707 \pm 3$ & $18,709.0$ & 4.7 & 4.7
\end{tabular}

\footnotetext{
${ }^{\text {a }}$ Values of molecular weight (MW) and isoelectric point (pI) measured by mass spectrometry and isoelectric focusing gel electrophoresis, respectively. Uncertainties for molecular weights were obtained from five to eight replicate measurements.

${ }^{\mathrm{b}}$ Values of MW and pI predicted using the program GPMAW (version 2.13a, Lighthouse Data).
} 


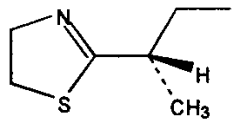

(S)-SBT<smiles>CCC(=O)CCC(C)(C)O</smiles>

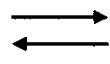

$\mathrm{HMH}$

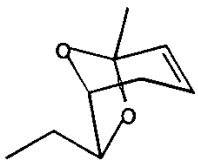

$(\mathrm{R}, \mathrm{R})-\mathrm{DHB}$

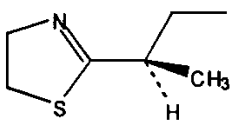

(R)-SBT
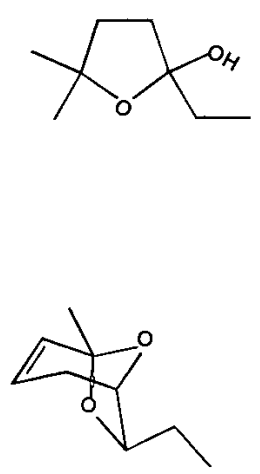

(S,S)-DHB
Fig. 1. Pheromonal ligands used in this study: $( \pm)-2$-sec-butyl-4,5-dihydrothiazole (SBT), 6-hydroxy-6-methyl-3-heptanone $(\mathrm{HMH})$, and $( \pm)$ dehydro-exo-brevicomin (DHB).

The X-ray structure of the MUP-I:HMH complex revealed that HMH binds in the open-chain form (Timm et al. 2001).

The binding affinities of MUP-ligand interactions were measured using isothermal titration calorimetry (ITC). Rep- resentative thermograms and fitted binding curves are presented in Figure 2 for a typical MUP-pheromone pair (MUP-I:SBT), the strongest-binding pair (MUP-IV:SBT), and the weakest-binding pair (MUP-IV:HMH). The dissociation constants $\left(\mathrm{K}_{\mathrm{d}}\right)$ determined for the various MUPpheromone complexes are listed in Table 2 and presented graphically in Figure 3. For all MUP-pheromone pairs studied, binding was exothermic $(\Delta \mathrm{H}$ in the range -6 to -20 $\mathrm{kcal} / \mathrm{mole}$ ), and the $\mathrm{K}_{\mathrm{d}}$ values (in the range $\sim 40 \mathrm{nM}$ to $\sim 200$ $\mu \mathrm{M})$ could be obtained with adequate precision by ITC. In all cases, the stoichiometry (n) determined from the ITC data was close to unity (in the range 0.80 to 1.35 ). A table of $\Delta \mathrm{H}$ and $\mathrm{n}$ values is presented in the Supplemental Material. A detailed analysis of the factors contributing to the observed enthalpy and entropy changes will be presented in a separate article. The $\mathrm{K}_{\mathrm{d}}$ values and stoichiometry obtained for MUP-I:SBT binding at $30^{\circ} \mathrm{C}\left(\mathrm{K}_{\mathrm{d}}=0.90 \pm 0.05 \mu \mathrm{M}\right.$ and $\mathrm{n}=0.80 \pm 0.02$ molecules of ligand per protein molecule) agree well with those determined previously from an equilibrium diffusion assay at $20^{\circ} \mathrm{C}\left(\mathrm{K}_{\mathrm{d}}=1.3 \pm 0.3 \mu \mathrm{M}\right.$ and $\mathrm{n}=1.0 \pm 0.1)$ and the stoichiometry determined by NMR titration at $30^{\circ} \mathrm{C}\left(\mathrm{n}=1.0 \pm 0.1\right.$; Zidek et al. 1999). The $\mathrm{K}_{\mathrm{d}}$ value determined for rMUP-I:DHB binding $\left(26 \mu \mathrm{M}\right.$ at $\left.30^{\circ} \mathrm{C}\right)$ also agrees well with the value reported previously for a natural MUP isolate $\left(20 \mu \mathrm{M}\right.$ at $25^{\circ} \mathrm{C}$; Bacchini et al. 1992).

We have corroborated the MUP-SBT binding affinities by monitoring the intrinsic fluorescence of MUP isoforms as a function of added SBT concentration. All six isoforms studied here have a single tryptophan residue that is located

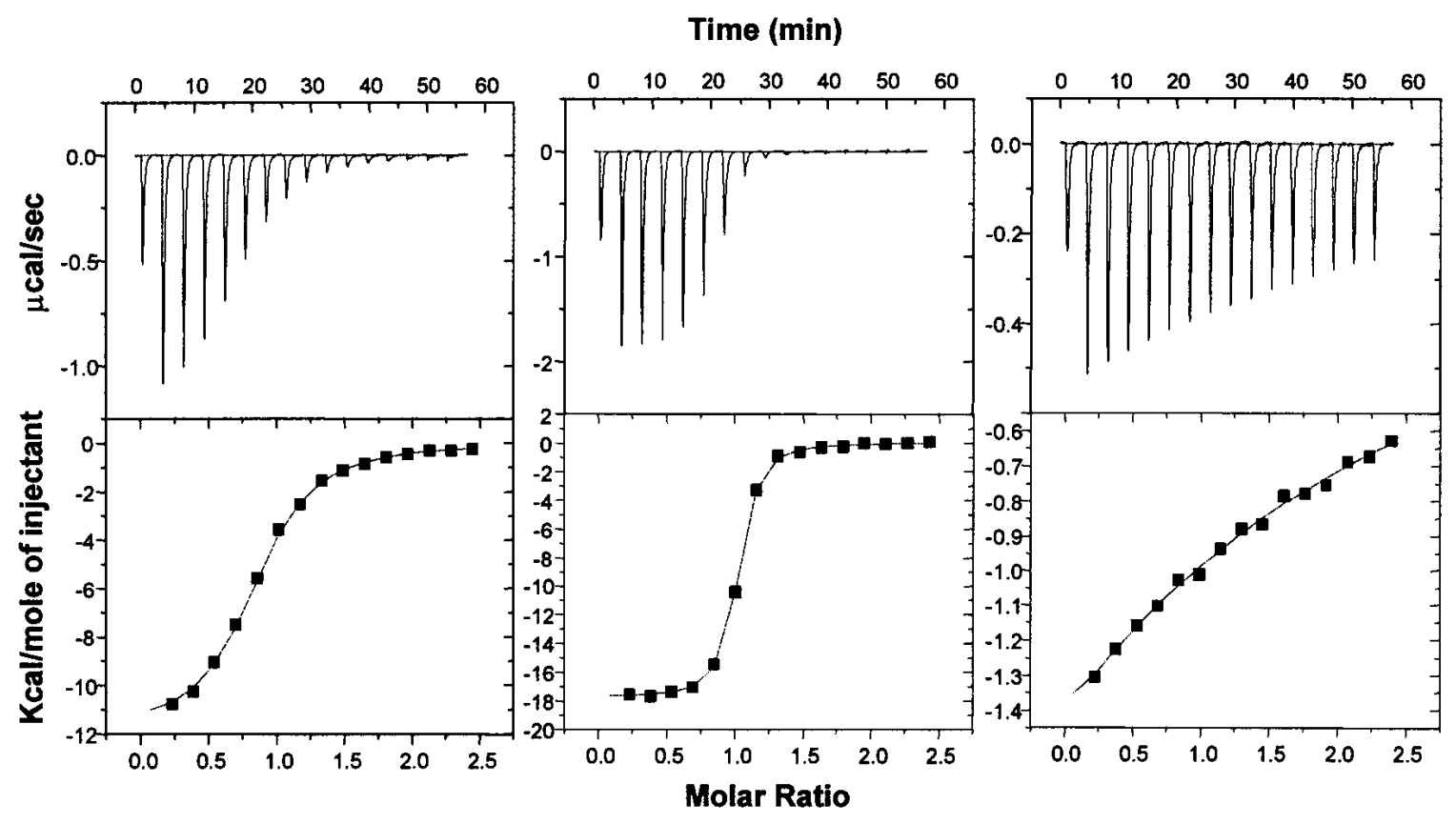

Fig. 2. Isothermal titration calorimetry data for binding of ( \pm )-2-sec-butyl-4,5-dihydrothiazole (SBT) to MUP-I (left), binding of SBT to MUP-IV (center), and binding of 6-hydroxy-6-methyl-3-heptanone (HMH) to MUP-IV (right) at $30^{\circ} \mathrm{C}$. Top panels are the raw data, and bottom panels are the fitted binding isotherms. Fitted $\mathrm{K}_{\mathrm{d}}$ values were 0.90, 0.039, and $199 \mu \mathrm{M}$, respectively. 
Table 2. MUP-pheromone binding affinities

\begin{tabular}{lccccrr}
\hline Isoform & $\begin{array}{c}\mathrm{K}_{\mathrm{d}}(\mathrm{SBT}) \\
(\mu \mathrm{M})\end{array}$ & $\begin{array}{c}\mathrm{K}_{\mathrm{d}}(\mathrm{SBT})^{\mathrm{a}} \\
(\mu \mathrm{M})\end{array}$ & $\begin{array}{c}\mathrm{K}_{\mathrm{d}}(\mathrm{HMH}) \\
(\mu \mathrm{M})\end{array}$ & $\begin{array}{c}\mathrm{K}_{\mathrm{d}}(\mathrm{DHB}) \\
(\mu \mathrm{M})\end{array}$ & $\mathrm{K}_{\mathrm{d}}(\mathrm{HMH}) / \mathrm{K}_{\mathrm{d}}(\mathrm{SBT})$ & $\mathrm{K}_{\mathrm{d}}(\mathrm{DHB}) / \mathrm{K}_{\mathrm{d}}(\mathrm{SBT})$ \\
\hline MUP-I & 0.90 & $2.5 \pm 1.9$ & 56 & 26 & 62 & 29 \\
MUP-II & 0.78 & $2.0 \pm 0.6$ & 54 & 29 & 69 & 37 \\
MUP-IV & 0.039 & $0.32 \pm 0.29$ & 199 & 58 & 5103 & 183 \\
MUP-VII & 0.56 & $0.29 \pm 0.12$ & 102 & 56 & 74 & 53 \\
MUP-VIII & 0.77 & $3.2 \pm 1.4$ & 57 & 31 & 66 & 40 \\
MUP-IX & 0.77 & $3.8 \pm 1.4$ & 51 & 31 &
\end{tabular}

Unless otherwise noted, $\mathrm{K}_{\mathrm{d}}$ values were determined by isothermal titration calorimetry. Uncertainties in these $\mathrm{K}_{\mathrm{d}}$ values are estimated to be $\sim 20 \%$, on the basis of triplicate and quadruplicate titrations recorded for MUP-I-SBT and MUP-IV-SBT binding, respectively. SBT indicates ( \pm )-2-sec-butyl-4,5dihydrothiazole; HMH, 6-hydroxy-6-methyl-3-heptanone; and DHB, ( \pm -dehydro-exo-brevicomin.

${ }^{a}$ These $\mathrm{K}_{\mathrm{d}}$ values were determined by fluorescence.

in the $\mathrm{N}$-terminal $\alpha$-helix, outside of the $\beta$-barrel into which the pheromone binds. However, there is a conserved tyrosine residue (Tyr-120) in the binding cavity that participates in a water-mediated hydrogen bond network between MUP-I and SBT (Timm et al. 2001); presumably, a similar network exists in the other MUP-SBT complexes. Consequently, the intrinsic tyrosine fluorescence is sensitive to MUP binding. Representative binding curves for both MUP-I:SBT and MUP-IV:SBT are shown in Figure 4, and the dissociation constants obtained by fluorescence are listed in Table 2; binding curves for the other isoforms are presented in Supplemental Material. The $\mathrm{K}_{\mathrm{d}}$ values determined by fluorescence show a similar trend to those determined by ITC in that SBT binds to MUP-IV and MUP-VII significantly more tightly than to the other MUP isoforms. As expected, the precision of the $\mathrm{K}_{\mathrm{d}}$ values derived from fluorescence is substantially lower than that of the values derived from ITC, owing to the small change in protein fluorescence on binding; thus, quantitative comparison of the data is difficult. Nevertheless, the observation of pheromone concentration-dependent changes in intrinsic MUP fluorescence is interesting because (1) it supports the role of the conserved tyrosine in pheromone binding, and (2) it raises the possibility of following binding kinetics using

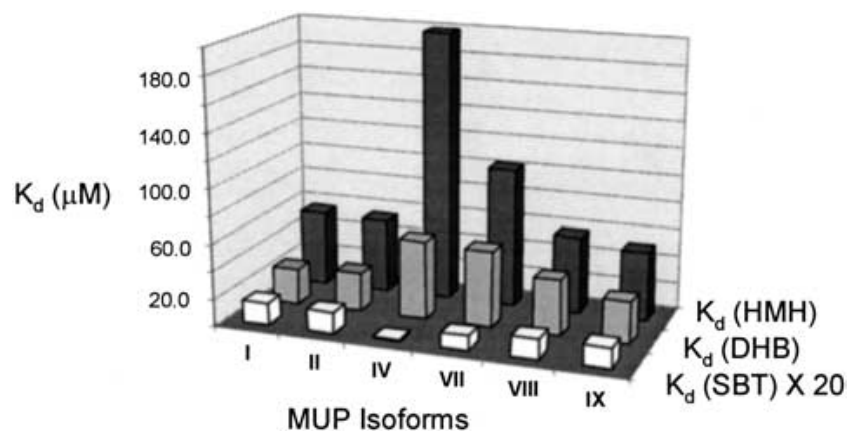

Fig. 3. Three-dimensional graphical representation of the measured dissociation constants for each MUP-pheromone pair. For ease of visual comparison, $\mathrm{K}_{\mathrm{d}}$ values for MUP-( \pm )-2-sec-butyl-4,5-dihydrothiazole (SBT) binding have been multiplied by a factor of 20 . stopped-flow fluorescence, as has been performed with similar proteins (Cistola et al. 1996; Richieri et al. 1999).

Comparison of the $\mathrm{K}_{\mathrm{d}}$ values (Table 2; Fig. 3) reveals several interesting trends. First, the affinity of each MUP isoform for SBT $\left(\mathrm{K}_{\mathrm{d}} \sim 0.04\right.$ to $\left.0.9 \mu \mathrm{M}\right)$ is higher than that for DHB $\left(\mathrm{K}_{\mathrm{d}} \sim 26\right.$ to $\left.58 \mu \mathrm{M}\right)$, which in turn is higher than

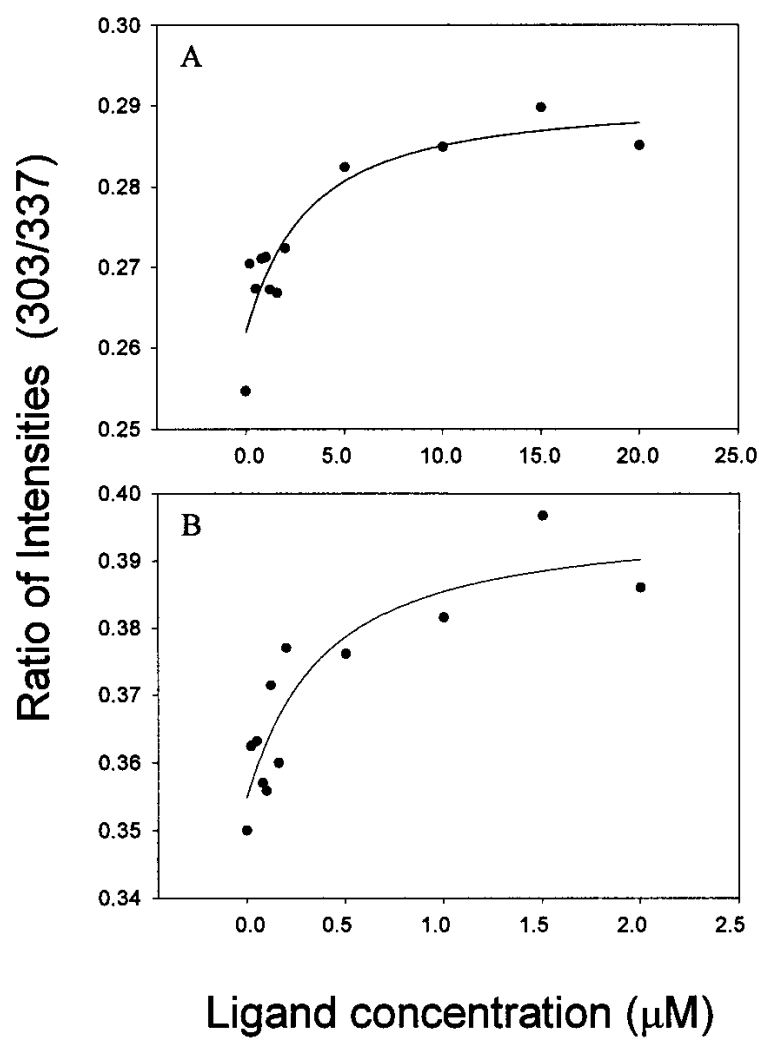

Fig. 4. Binding of $( \pm$ )-2-sec-butyl-4,5-dihydrothiazole (SBT) to MUP-I $(A)$ and MUP-IV $(B)$, determined by intrinsic protein fluorescence. Each curve shows the ratio of fluorescence emission intensities at wavelengths of 303 and $337 \mathrm{~nm}$ plotted against the total SBT concentration. Protein concentrations were $1.0 \mu \mathrm{M}$ (MUP-I) and $0.1 \mu \mathrm{M}$ (MUP-IV), and the excitation wavelength was $228 \mathrm{~nm}$. The fitted binding isotherms yielded $\mathrm{K}_{\mathrm{d}}$ values of $2.5 \pm 1.9$ and $0.32 \pm 0.29 \mu \mathrm{M}$, respectively. Note that the $\mathrm{x}$-axis scale differs by a factor of 10 between the two panels. 
that for HMH ( $\mathrm{K}_{\mathrm{d}} \sim 50$ to $\left.200 \mu \mathrm{M}\right)$. Second, MUP isoforms I, II, VIII, and IX show very similar $\mathrm{K}_{\mathrm{d}}$ values for each of the ligands ( 0.8 to $0.9 \mu \mathrm{M}$ for SBT, 26 to $41 \mu \mathrm{M}$ for DHB, and 50 to $57 \mu \mathrm{M}$ for HMH). Third, MUP-IV and MUP-VII show higher affinity than the other isoforms for SBT, but lower affinity for DHB and HMH; the affinity differences are particularly dramatic for MUP-IV, which binds $\sim 23$-fold more tightly than MUP-I to SBT. The variations of $K_{d}$ values reveal a substantial difference in ligand specificity among the different isoforms. Thus, MUP-I shows a $\sim 62-$ fold preference for SBT over HMH, whereas MUP-IV shows a $~ 5100$-fold preference.

The differential binding affinities shown by MUP-IV are consistent with MUP-IV having substantial sequence differences from the other isoforms (see sequence alignment, Fig. 5), and with MUP-IV being the only isoform studied here that is not expressed in the urine and for which mRNA has been identified in the oral sphere organs (Shahan et al. 1987); additional lipocalins have been found previously in the nasal glands (Pes and Pelosi 1995; Utsumi et al. 1999). To investigate whether MUP-IV protein is indeed expressed in the nasal cavity, we performed chromatographic analyses of proteins extracted from murine nasal mucus and from murine vomeronasal tissue. After a two-step fractionation, both extracts yielded samples of MUP-IV protein, which was identified on the basis of the following observations: (1) the molecular mass was measured to be $18,817 \mathrm{Da}$, identical to the mass predicted from the cDNA sequence; (2) the isoelectric point was 5.1, close to the value of 5.3 determined for recombinant MUP-IV; (3) the N-terminal amino acid sequence was determined to be EEATSKGQN, as predicted for MUP-IV (underlined in Fig. 5); (4) the masses of three internal tryptic fragments $(1131.8,1257.0$, and $1748.0 \mathrm{Da}$ ) were consistent with regions of the predicted MUP-IV sequence (bold in Fig. 5); and (5) mass- spectrometric postsource decay analysis of the 1257.0-Da peptide yielded the sequence TFQLMELYGR, consistent with an internal portion of MUP-IV (bold and underlined in Fig. 5). None of the predicted amino acid sequences for other known MUP isoforms or murine odorant binding proteins is consistent with all of the above data. However, nasal extracts also yielded evidence for the two odorant-binding proteins, OBP-I and OBP-II (data not shown; Zidek 1999).

\section{Discussion}

\section{MUP isoform classification}

In the current study, we have compared the binding affinities of six MUP isoforms to three pheromones. The amino acid sequences of the six MUP isoforms are aligned in Figure 5. Al-Shawi et al. (1989) have classified MUP isoforms into four groups on the basis of patterns of expression and posttranslational modification. Five of the isoforms studied here (MUPs-I, II, VII, VIII, and IX) belong to group 1 , which comprises nonglycosylated isoforms expressed in the liver under multiple hormone control and subsequently filtered into the urine. These five isoforms share $98.1 \%$ to 99.4\% sequence identity. The other isoform studied here, MUP-IV, belongs to group 4, which comprises MUP isoforms synthesized in lachrymal and salivary glands, but not the liver. MUP-IV shares only $74.1 \%$ to $75.3 \%$ identity with the group 1 isoforms studied herein. Group 2 isoforms are pseudogenes, apparently not expressed because of inappropriate stop codons and frame-shifts. Finally, group 3 includes isoforms that are synthesized in the liver and excreted into the urine, but differ from group 1 isoforms in that they are glycosylated and are under slightly different hormonal control. In the discussion that follows, we address the structural basis of the affinity differences observed here and

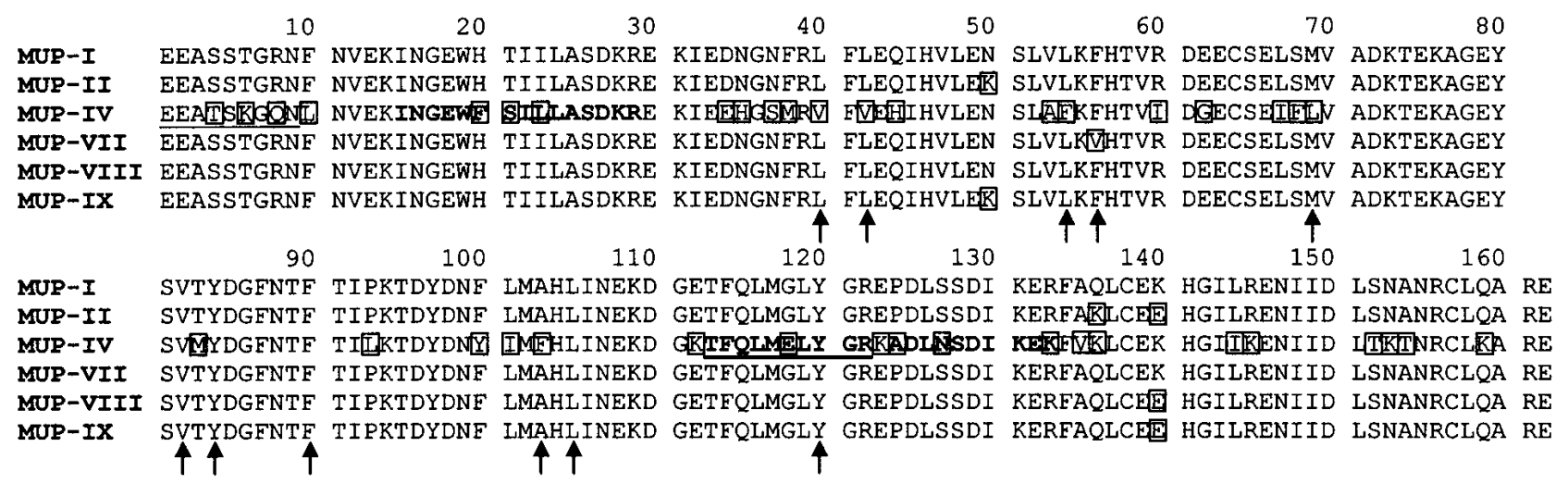

Fig. 5. Sequence alignment of the six MUP isoforms included in this study. Amino acids that differ from the corresponding residue in MUP-I are boxed. Positions of MUP-I residues with side-chains that show NOEs to ( \pm )-2-sec-butyl-4,5-dihydrothiazole (SBT; Zidek et al. 1999) are indicated by arrows. The N-terminal sequence determined by Edman sequencing of nasal MUP-IV is underlined. Internal MUP-IV tryptic fragments observed by mass spectrometry are indicated in bold; the internal fragment sequenced by massspectrometric postsource decay analysis is also underlined. 
the implications of the binding data for the biological roles of both urinary (group 1) and nasal (group 4) MUP isoforms.

\section{Correlation of affinity with isoform structure}

The MUP-pheromone binding affinities determined here can be interpreted in light of the available structural data. $\mathrm{X}$-ray crystal structures have been reported recently for recombinant MUP-I bound to SBT and HMH (Timm et al. 2001). In addition, we have characterized the interactions between SBT and MUP-I in solution using NMR spectroscopy (Zidek et al. 1999), and an NMR structure of recombinant MUP-II has also been reported (Lucke et al. 1999). X-ray structures of MUPs isolated from urine (Bocskei et al. 1992) and liver (Timm et al. 2001) are also available. MUPs consist of an eight-stranded antiparallel $\beta$-sheet with +1 connections (i.e., a $\beta$-meander). The terminal strands are hydrogen-bonded to each other, so that the sheet forms a closed $\beta$-barrel ( $\beta$-clam structure). The ends of the barrel are capped by a short $3_{10}$-helix and by an $\Omega$-loop, and the interior of the barrel forms a predominantly hydrophobic cavity, which is the binding site for ligands. The final secondary structural feature is a C-terminal $\alpha$-helix, which packs against the outside of the $\beta$-barrel. NMR data revealed 11 nonpolar amino acid side-chains lining the MUP-I binding pocket that have NOE contacts with SBT; these are indicated by arrows in Figure 5. Among the isoforms studied here, these 11 residues are identical in MUPs-I, II, VIII, and IX, consistent with the nearly identical $\mathrm{K}_{\mathrm{d}}$ values shown by these isoforms for all three pheromonal ligands. MUPVII has a single-amino-acid replacement in the binding cavity (Phe-56 $\rightarrow$ Val) relative to MUP-I. Thus, this conservative substitution appears to be responsible for the subtle (approximately twofold) increase in affinity for SBT and decreases in affinity for HMH and DHB (Table 2). Consistent with these observations, substitution of Phe-56 by valine results in a decrease in the affinity of MUP-I for the fluorescent probe $N$-phenyl-naphthylamine (Marie et al. 2000). Finally, MUP-IV has substitutions relative to MUP-I at five of the contact residues (Leu-40 $\rightarrow \mathrm{Val}$, Leu-42 $\rightarrow \mathrm{Val}$, Leu-54 $\rightarrow$ Phe, Met-69 $\rightarrow$ Leu, and Ala-103 $\rightarrow$ Phe). Notably, all of these mutations involve replacement of one hydrophobic residue by another, although the Ala- $103 \rightarrow$ Phe substitution involves a substantial increase in side-chain size. Although, there are 35 other substitutions in MUP-IV relative to MUP-I, it seems likely that the differences in affinity observed here are attributable predominantly to the five cavity mutations.

\section{Possible roles of hydrogen bonding} and hydrophobic interactions

In the crystal structures of MUP-I bound to SBT and HMH, there is a conserved, water-mediated hydrogen bonding net- work between the ligand and the protein (Timm et al. 2001). In the SBT complex, the SBT ring nitrogen hydrogen-bonds to an ordered water molecule, which is also hydrogenbonded to a second water and to the backbone carbonyl group of Phe-56. The second water molecule is hydrogenbonded in turn to the backbone carbonyl group of Leu-58 and the phenolic hydroxyl group of Tyr-120. The Leu-58 $\mathrm{CO}$ group is also hydrogen-bonded to the side-chain of Thr21 , possibly helping to position it appropriately for optimal hydrogen-bonding to the second water. In the HMH complex, two water molecules are observed, making identical interactions with the protein. However, the first water molecule is hydrogen-bonded to the ligand carbonyl group, and the ligand hydroxyl group donates a hydrogen bond to the Tyr-120 hydroxyl group of MUP-I. Taken together, these observations indicated that the hydrogen bond network may make a substantial energetic contribution to binding of MUP-I to both SBT and HMH (Timm et al. 2001). However, the current data indicate that substitutions of the hydrophobic side-chains in the binding cavity are sufficient to alter the binding affinity by up to $\sim 23$-fold (MUP-I to MUPIV). Furthermore, an $\sim 60$-fold affinity difference is observed for binding of MUP-I to SBT and HMH, even though similar hydrogen bond networks are formed in both interactions. Thus, it appears either that nonpolar interactions can also contribute significantly to the free energy of MUPpheromone binding or that alterations of the hydrophobic side-chains can modulate the strengths of the water-mediated hydrogen bonds; the latter effect could occur if sidechain substitutions alter the position of the ligand in the binding cavity.

\section{Regulation of pheromone levels by urinary MUPs}

There is evidence that one of the biological roles of urinary MUPs is to regulate the rate of pheromone release by controlling the concentrations of free (volatile) pheromones in urine. We have used the current thermodynamic data and previous data on the typical urinary concentrations of pheromones and MUPs to estimate the concentrations of free pheromones, free MUP isoforms, and MUP-isoform complexes in mouse urine. These calculations were performed for total MUP concentrations of 55 to $264 \mu \mathrm{M}$ ( 1 to 5 $\mathrm{mg} / \mathrm{mL}$; Ferrari et al. 1997), and total pheromone concentrations of $9.4 \mu \mathrm{M}$ (1.3 ppm) SBT (Novotny et al. 1985), 8.4 $\mu \mathrm{M}$ (1.3 ppm) DHB (Novotny et al. 1985), and 3.4 to 10.0 $\mathrm{mM}$ (613 to $1819 \mathrm{ppm}$ ) HMH (Novotny et al. 1999a). Consensus $\mathrm{K}_{\mathrm{d}}$ values of $0.90 \mu \mathrm{M}$ (MUP-SBT), $26.2 \mu \mathrm{M}$ (MUPDHB), and 55.6 $\mu \mathrm{M}$ (MUP-HMH) were used because the $\mathrm{K}_{\mathrm{d}}$ values determined herein varied by less than approximately twofold among the urinary MUP isoforms.

The calculations indicated that (for an $\mathrm{HMH}$ concentration of $3.4 \mathrm{mM}$ ) increasing the total MUP concentration from 1.0 to $5.0 \mathrm{mg} / \mathrm{mL}$ decreases the concentration of free 
SBT from $3.6 \mu \mathrm{M}$ (38\% of total SBT) to $1.5 \mu \mathrm{M}$ (16\% of total SBT). This result indicates that variations of MUP concentrations within the normal physiological range can indeed regulate the concentration of available SBT. On the other hand, increasing the total MUP concentration from 1.0 to $5.0 \mathrm{mg} / \mathrm{mL}$ does not substantially influence the concentrations of available HMH or DHB. The concentration of free $\mathrm{HMH}$ is reduced from 3.3 to $3.2 \mathrm{mM}$ (98\% to $93 \%$ of total $\mathrm{HMH}$ ); variation of total MUP concentration is not an effective strategy for controlling the concentration of free $\mathrm{HMH}$, because the total $\mathrm{HMH}$ concentration exceeds the total MUP concentration by at least an order of magnitude. The fivefold increase of total MUP concentration reduces the concentration of free DHB from 8.1 to $7.1 \mu \mathrm{M}$ (97\% to $85 \%$ of total DHB). Although this corresponds to a 4.3 -fold increase in the concentration of MUP-DHB complexes, the total MUP concentration of $5 \mathrm{mg} / \mathrm{mL}$ remains insufficient to bind more than $\sim 15 \%$ of the total DHB present.

The calculations also reveal the potential importance of competitive binding in controlling the concentrations of free pheromones. At a total MUP concentration of $5 \mathrm{mg} / \mathrm{mL}$, increasing the total $\mathrm{HMH}$ concentration from 3.4 to 10.0 $\mathrm{mM}$ causes the concentration of free SBT to increase from $1.9 \mu \mathrm{M}(20 \%$ of total SBT) to $5.5 \mu \mathrm{M}$ (58\% of total SBT). Thus, the concentrations of available pheromones may be influenced by changes in the expression levels of urinary MUPs or by changes in the excretion levels of other pheromones (or, presumably, nonpheromonal MUP ligands).

\section{Biological role of urinary MUP heterogeneity}

The biological relevance of heterogeneity among urinary MUPs remains poorly defined. It has been proposed that variation of MUP profiles gives rise to differential binding and release of pheromones in different strains or individuals or at different times in a single individual (Robertson et al. 1996). This proposal requires that there are significant variations in pheromone affinity between MUP isoforms. In the current study, we have found that MUP-VII shows subtle differences in SBT, HMH, and DHB affinity relative to the other urinary MUPs studied. Furthermore, MUP-VII has an approximately fourfold increase in specificity for SBT over the other pheromones, providing some support for the proposal that variations in MUP profiles could regulate the profiles of volatile pheromones. We have found here that the four isoforms MUP-I, II, VIII, and IX show almost identical affinities for all three pheromones studied. Thus, the theory that the function of MUP heterogeneity is to differentially regulate pheromone levels is not sufficient to explain the expression of these four MUP isoforms. However, there are apparently many additional MUPs that could be tested, and Marie et al. (2000) have noted that the role of MUPs in ligand release indicates that the polymorphisms may lead to different binding characteristics for molecules that occupy the central calyx.

There are several alternative possible biological roles for urinary MUP heterogeneity. First, it has been speculated that the various MUP isoforms expressed by one individual may be directly detected by a second individual, providing a mechanism for the recognition of members of the same strain or family or of potential mates (Cavaggioni et al. 1999). A very recent paper (Hurst et al. 2001b) is supportive of this hypothesis. Second, the MUP isoforms and/or their pheromone complexes may bind directly to VNO or olfactory receptors, leading to modulation of pheromonal signals depending on the profile of MUP isoforms. A third possibility is that heterogeneity is required merely to prevent crystallization of MUP protein in the urine or the kidneys; the total urinary MUP concentration of $\sim 1$ to $5 \mathrm{mg} / \mathrm{mL}$ is close to the concentration used to crystallize MUP-I and its complexes (Ferrari et al. 1997). Any of these three possibilities requires differences between the surface structural elements of the MUP isoforms. Thus, these proposals are consistent with the differences in surface amino acids between MUPs-I, II, VIII, and IX. On the other hand, they would not be sufficient to explain the structural difference between MUP-VII and these other urinary isoforms (this difference is localized to the interior binding cavity). Taken together, these observations lead to the possibility that MUP heterogeneity has more than one biological role: Variations in surface residues may affect direct MUP recognition or self-association, whereas variations in the binding cavity may influence the profile of available pheromones.

\section{Biological function of nasal MUPs}

Among the MUP isoforms studied here, MUP-IV is the only one expressed in glands near the nasal cavity. Gene hybridization studies have identified MUP-IV mRNA in the lachrymal gland (Shahan et al. 1987), and we have now found evidence for MUP-IV protein in the nasal mucus and VNO. Furthermore, we have found that the pheromone specificity of MUP-IV differs dramatically from those of the urinary isoforms studied here. The $\sim 25$-fold higher affinity of MUPIV for SBT indicates that a large fraction of the SBT that may enter the nasal cavity bound to one of the urinary MUPs is likely to dissociate from the urinary MUP and then associate with MUP-IV. This will be true as long as the concentration of MUP-IV is comparable to or higher than the concentration of urinary MUP in the nasal cavity (most likely the case, because none of the urinary MUPs is expressed naturally in the nasal region), and as long as the dissociation rate from urinary MUPs is sufficiently fast to allow transfer to nasal MUPs. The latter criterion appears to be satisfied; the dissociation rate of the MUP-SBT complex is on the order of a few seconds (J.L. Vaughn, M.V. Novotny, and M.J. Stone, unpubl.). In addition to sequestering 
SBT from urinary MUPs, MUP-IV may also "capture" free SBT that has diffused into the nasal cavity. Thus, it appears likely that MUP-IV serves to concentrate SBT in nasal mucus and may also transport SBT into the VNO, where it can associate with its neuronal receptor(s). On the other hand, MUP-IV binds much more weakly to HMH and DHB than to SBT, and both of these other pheromones show weak ( $\sim$ two- to fourfold) preferences for urinary MUPs relative to MUP-IV. Again, if HMH and DHB enter the nasal cavity complexed to urinary MUPs, they will be transferred to MUP-IV if the concentration of MUP-IV in the nasal cavity is substantially higher than that of urinary MUPs in the nasal cavity.

\section{Summary}

We have determined the affinities of five urinary MUP isoforms and one nasal MUP isoform for three natural pheromonal ligands. The variations in affinities are consistent with structural differences in the binding cavities of the isoforms and indicate an important role for hydrophobic interactions in controlling affinity and specificity. The data indicate that the concentrations of available pheromones may be influenced by the expression levels of urinary MUPs or by changes in the excretion levels of other MUP ligands. However, the variations in pheromone affinities of the urinary MUP isoforms provides only limited support for the proposal that MUP heterogeneity plays a role in regulating profiles of available pheromones. The existence of several isoforms that differ only at positions on the surface of the protein (not in the binding cavity) indicates several alternative (or additional) possible roles for MUP heterogeneity. On the other hand, dramatic affinity and specificity differences between nasal MUP-IV and the urinary isoforms indicate that nasal MUPs play a role in sequestering pheromones (particularly SBT) and possibly in transporting them to vomeronasal neurons.

\section{Materials and methods}

\section{MUP expression and purification}

Expression vectors for MUP-II, MUP-VII, MUP-VIII, and MUPIX were obtained by Quikchange (Stratagene) mutagenesis of the MUP-I gene contained in a pET28 (Novagen) expression vector. A gene encoding MUP-IV, with codons optimized for expression in E. coli, was synthesized from 10 overlapping oligonucleotides using recursive PCR (Prodromu and Pearl 1992) and then subcloned between the XhoI and NcoI restriction sites of the plasmid pET28b(+) (Novagen). Each expression vector encodes a His ${ }_{6}$-tag to facilitate affinity-purification and a factor Xa cleavage site, followed by the amino acid sequence of the relevant MUP protein. MUP isoforms were expressed and purified as described (Zidek et al. 1999), with the minor modification that the nickel-affinity (HisBind, Novagen) chromatographic step was implemented a second time on the factor Xa cleavage products to remove uncleaved fusion protein from cleaved protein.

\section{Protein characterization}

Isoelectric focusing gel electrophoresis was performed using a Bio-Phoresis horizontal electrophoresis cell. A T5C3 acrylamide gel was formed using Biolyte 4/6 and 3/10 ampholites resulting in a $\mathrm{pH}$ gradient of 4 to 10 . Approximately $10 \mu \mathrm{g}$ of sample was loaded per lane. The gel was run for approximately $2 \mathrm{~h}$ at $1000 \mathrm{~V}$ and $25^{\circ} \mathrm{C}$, then visualized by Coomassie stain. An isoelectric focusing gel illustrating the purity of the recombinants MUP isoforms is included in the Supplemental Material. Each sample displayed a single predominant band. A few faint bands (mostly undetectable by matrix-assisted laser desorption ionization, timeof-flight (MALDI-TOF) mass spectrometry) were also visible. These trace populations were not characterized further, as they were not expected to contribute significantly to equilibrium binding measurements.

Mass analysis was performed using a PerSeptive Biosystems Voyager-DE RP MALDI-TOF mass spectrometer equipped with a nitrogen laser $(337 \mathrm{~nm})$. The spectra was collected in the linear mode, with an accelerating voltage of $25,000 \mathrm{~V}$, grid voltage at $89.9 \%$, guide-wire voltage of $0.05 \%$, and pulse delay of $175 \mathrm{nsec}$. The low-mass gate was selected at 1500 , and the laser intensity was set at 2370. Spectra were calibrated internally with both horse skeletal apomyoglobin and trypsin inhibitor. Both the analyte (10 pmole) and the calibrants were mixed in $10 \mathrm{mg} / \mathrm{mL} 3,5$-dimethyl4-hydroxy-cinnamic acid. Signal-averaged mass spectra for 64 laser pulses were collected, and data were analyzed using Grams/386 software. Eight to 10 independent measurements were taken for statistical analysis.

\section{Ligand synthesis}

SBT, HMH, and DHB were prepared using published methods (Wasserman and Oku 1986; North and Pattenden 1990; Novotny et al. 1999a; Zidek et al. 1999). Structures were verified by ${ }^{1}$ H NMR and mass spectrometry. Purity was determined by gas chromatography-mass spectrometry; all samples were $>97 \%$ pure.

\section{Isothermal titration calorimetry}

Isothermal titration calorimetry was performed using a Microcal MCS instrument located at the Keck Biophysics Facility, Northwestern University, Evanston, IL. Samples of MUP protein (2.4 $\mathrm{mL}$ of 13 to $500 \mu \mathrm{M}$ protein in $10 \mathrm{mM}$ phosphate, $0.02 \% \mathrm{NaN}_{3}$ at $\mathrm{pH}$ 6.3) were titrated with one $2-\mu \mathrm{L}$ aliquot, then fifteen $4-\mu \mathrm{L}$ aliquots of pheromone ( 0.01 to $2.6 \mathrm{mM}$ in the same buffer). The duration of each injection was $19.76 \mathrm{sec}$, with a 210 -sec recovery time between injections. All titrations were performed at $30^{\circ} \mathrm{C}$. The reference cell was filled with distilled water. To account for heats of dilution and mechanical mixing, control titrations were performed by injection of ligand into buffer; binding data were corrected by subtraction of dilution data before curve fitting. Corrected binding data were processed using the Origin ITC analysis software package supplied by Microcal (Northampton, MA). The data were fit well by the following equation describing the cumulative heat $(\mathrm{Q})$ evolved from a series of injections when the ligand (L) binds to a set of identical, independent binding sites on the protein. 


$$
\begin{aligned}
Q= & \left(1+[P]_{\text {total }} n K_{a}+K_{a}[L]_{\text {total }}\right) \\
& -\left\{\sqrt{\begin{array}{c}
\left(1+[P]_{\text {total }} n K_{a}+K_{a}[L]_{\text {total }}\right)^{2} \\
-4[P]_{\text {total }} n K_{a}^{2}[L]_{\text {total }}
\end{array}} \frac{2 K_{a}}{V \Delta H^{0}}\right\}
\end{aligned}
$$

$\mathrm{V}$ represents the initial reaction volume. Nonlinear curve fitting of the first derivative of $\mathrm{Q}$ with respect to $[\mathrm{L}]_{\text {total }}$ plotted against the molar ratio of $\left([\mathrm{L}]_{\text {total }} /[\mathrm{M}]_{\text {total }}\right)$ yielded the association equilibrium constant $\left(\mathrm{K}_{\mathrm{a}}\right)$, the binding stoichiometry $(\mathrm{n}=$ number of ligand binding sites per protein molecule), and the enthalpy of binding $\left(\Delta \mathrm{H}^{0}\right)$

\section{Fluorescence spectroscopy}

Fluorescence experiments were performed on a Perkin-Elmer LS50B luminescence spectrometer using 1-mL quartz fluorescence sample cells. The temperature was controlled with a Neslab RTE140 circulating water bath set at $30^{\circ} \mathrm{C}$. Samples contained MUP protein $(0.1 \mu \mathrm{M}$ for MUP-IV and $1.0 \mu \mathrm{M}$ for all other isoforms), SBT (0.02 to $2.0 \mu \mathrm{M}$ for MUP-IV and 0.20 to $20 \mu \mathrm{M}$ for all other isoforms) in $10 \mathrm{mM}$ phosphate buffer ( $\mathrm{pH}$ 6.3). Fluorescence emission intensities were determined at $303 \mathrm{~nm}$ and $337 \mathrm{~nm}$ using an excitation wavelength of $228 \mathrm{~nm}$, corresponding to the ${ }^{1} \mathrm{~L}_{\mathrm{a}}$ transition of the tyrosine aromatic side-chain (Ross et al. 1992). For most isoforms, the ratio of emission intensities at $303 \mathrm{~nm}$ and 337 $\mathrm{nm}$ was fit as a function of SBT concentration; the ratio mode was chosen to minimize errors owing to small differences in protein concentration. For MUP-VII binding data, the ratio data were noisy because of similar spectral changes at both wavelengths; therefore, the emission data at a single wavelength $(337 \mathrm{~nm})$ were fit as a function of SBT concentration for this isoform (see Supplemental Material). In either case, binding data were fit using SigmaPlot (version 4.01, SPSS Inc.) to the equation:

$$
y=0.5\left(K_{d}+P_{t}+L_{t}\right)+\left[\left(K_{d}+P_{t}+L_{t}\right)^{2}-\left(4 P_{t} L_{t}\right)\right]^{1 / 2}
$$

in which $\mathrm{y}$ is the emission intensity or intensity ratio (see above), $\mathrm{P}_{\mathrm{t}}$ is the known total concentration of protein, $\mathrm{L}_{\mathrm{t}}$ is the known total concentration of ligand, and $\mathrm{K}_{\mathrm{d}}$ is the fitted equilibrium dissociation constant.

\section{Isolation and analysis of MUP-IV from murine nasal тисиs and vomeronasal organ}

Nasal mucus was collected from anesthetized ICR/Alb mice treated with isoproterenol. Vomeronasal organs of killed animals were removed, and the fluid was extracted with an excess of 10 $\mathrm{mM}$ Tris buffer ( $\mathrm{pH}$ 7.8). Both the nasal mucus and VNO fluids were exchanged into $10 \mathrm{mM}$ Tris buffer $(\mathrm{pH} 7.8)$ by filtration in Amicon Microcon 10 microconcentrators under a centrifugal force of $3300 \mathrm{~g}$. For each starting material, the crude isolate was loaded onto a Pharmacia anion-exchange miniQ PC 3.2/3 column in 50 $\mu \mathrm{L}$ aliquots and eluted with a 0 to $0.5 \mathrm{M} \mathrm{NaCl}$ gradient at a flow rate of $0.2 \mathrm{~mL} / \mathrm{min}$ for $15 \mathrm{~min}$. The first fraction to show binding activity (see below) was exchanged into $10 \mathrm{mM}$ sodium phosphate buffer and $1.8 \mathrm{M}$ ammonium sulfate $(\mathrm{pH}$ 6.8) and loaded onto a Pharmacia Superose PC 1.6/5 hydrophobic-interaction phenyl column at $50 \mu \mathrm{L} / \mathrm{min}$. The material was eluted with a gradient of 10 $\mathrm{mM}$ sodium phosphate buffer and $1.8 \mathrm{M}$ ammonium sulfate $(\mathrm{pH}$ 6.8 ) to $10 \mathrm{mM}$ sodium phosphate and $20 \%$ ethylene glycol (pH 6.8), at a flow rate of $50 \mu \mathrm{L} / \mathrm{min}$ for $15 \mathrm{~min}$. Binding of SBT to purified MUP-IV, or chromatographic fractions, was measured by adding $0.1 \mathrm{mM}$ pheromone to $0.1 \mathrm{~mL}$ MUP samples. The samples were incubated for $30 \mathrm{~min}$ and filtered to dryness in Microcon 10 microconcentrators, washed with $20 \mu \mathrm{L}$ water, and filtered again. Following a dilution in $100 \mu \mathrm{L}$ water, the protein fraction was extracted with $20 \mu \mathrm{L} n$-heptane in a glass microvial; $6 \mu \mathrm{L}$ of the organic layer, separated by brief centrifugation, was analyzed by gas chromatography, as described (Zidek 1999). N-terminal protein sequencing was performed by automated Edman degradation on a Model 477A sequencer from PE Biosystems using the standard manufacturer's protocol. Partial sequencing of tryptic peptides was achieved mass-spectrometrically using postsource decay analysis.

\section{Acknowledgments}

We thank Dr. Patrick Koren, Michael Mayer, and Dr. Kristen Mayer, for helpful discussions, as well as Michael Goodman and Kate Edmonds for technical assistance. We would especially like to thank Dr. Katharina Spiegel for numerous helpful discussions regarding ITC and appreciate the access to the ITC instrument provided by the Keck Biophysics Facility at Northwestern University. This work was supported by grants awarded to M.V.N. from the National Institute of Deafness and Communication Disorders (DC 02418) and to M.J.S. from the National Science Foundation (MCB-9600968).

The publication costs of this article were defrayed in part by payment of page charges. This article must therefore be hereby marked "advertisement" in accordance with 18 USC section 1734 solely to indicate this fact.

\section{References}

Al-Shawi, R., Ghazal, P., Clark, A.J., and Bishop, J.O. 1989. Intraspecific evolution of a gene family coding for urinary proteins. J. Mol. Evol. 29: 302-313.

Bacchini, A., Gaetani, E., and Cavaggioni, A. 1992. Pheromone binding-proteins of the mouse, Mus-musculus. Experientia 48: 419-421.

Beynon, R., Robertson, D., Hubbard, S.J., Gaskell, S.J., and Hurst, J.L. 1999. The role of protein binding in chemical communication: Major urinary proteins in the house mouse. Advances in chemical communications in vertebrates (ed. R.E. Johnston), pp. 137-147. New York, Plenum Press.

Bocskei, Z., Groon, C.R., Flower, D.R., Wright, C.E., Phillips, S.E.V., Cavaggioni, A., Findlay, J.B.C., and North, A.C.T. 1992. Pheromone binding to two rodent urinary proteins revealed by X-ray crystallography. Nature 360: 186-189.

Buck, L. and Axel, R. 1991. A multigene family may encode odorant receptors: A molecular basis for odor recognition. Cell 65: 175-187.

Cavaggioni, A., Mucignat-Caretta, C., and Tirindelli, R. 1999. Pheromone signalling in the mouse: Role of urinary proteins and vomeronasal organ. Arch. Ital. Biol. 137: 193-200.

Cistola, D.P., Kim, K., Rogl H., and Frieden, C. 1996. Fatty acid interactions with a helix-less variant of intestinal fatty acid-binding protein. Biochemistry 35: 7559-7565.

Clissold, P.M and Bishop, J.O. 1982. Variation in mouse major urinary protein (MUP) genes and the MUP gene products within and between inbred lines. Gene 18: 211-220.

Dulac, C. and Axel, R. 1995. A novel family of genes encoding putative pheromone receptors in mammals. Cell 83: 195-206.

Ferrari, E., Lodi, T., Sorbi, R.T., Tirindelli, R., Cavaggioni, A., and Spisni, A. 1997. Expression of a lipocalin in pichia pastoris: Secretion, purification and binding activity of a recombinant mouse major urinary protein. FEBS Lett. 401: 73-77.

Finlayson, J.S. and Baumann, C.A. 1958. Mouse proteinuria. Am. J. Physiol. 192: $69-72$

Hurst, J.L., Robertson, D.H.L., and Beynon, R.J. 1998. Proteins in urine scent marks of male house mice extend the longevity of olfactory signals. Anim. Behav. 55: 1289-1297.

Hurst, J.L., Beynon, R.J., Humphries, R., Malone, N., Nevison, C.M., Payne, C.E., Robertson, D.H.L., and Veggerby, C. 2001a. Information in scent 
signals of competitive social status: The interface between behaviour and chemistry. In Chemical signals in vertebrates 9 (eds. A. Marchlewska-Koj, et al.), pp. 43-52. Kluwer Academic/Plenum Publishers, New York.

Hurst, J.L., Payne, C.E., Nevison, C.M., Amr, D.M., Humphries, R.E., Robertson, D.H.L., Cavaggioni, A., and Beynon, R.J. 2001b. Individual recognition in mice mediated by major urinary proteins. Nature 414: 631-634.

Jemiolo, B. and Novotny, M. 1994. Inhibition of sexual maturation in juvenile male and female mice by a chemosignal of female origin. Physiol. Behav. 55: 519-522.

Jemiolo, B., Harvey, S., and Novotny, M. 1986. Promotion of the Whitten effect in female mice by the synthetic analogs of male urinary constituents. Proc. Natl. Acad. Sci. 83: 4576-4579.

Johnson, D., Al-Shawi, R., and Bishop, J.O. 1995. Sexual dimorphism and growth hormone induction of murine pheromone-binding proteins. J. Mol. Endocrinol. 14: 21-34.

Lucke, C., Franzoni, L., Abbate, F., Lohr, F., Ferrari, E., Sorbi, R.T, Ruterjans, H., and Spisni, A. 1999. Solution structure of a recombinant mouse major urinary protein. Eur. J. Biochem. 266: 1210-1218.

Marie, A.D., Veggerby, C., Robertson, D., Gaskell, S.J., Hubbard, S.J., Martinsen, L., Hurst, J.L., and Beynon, R. 2000. Effect of polymorphisms on ligand binding by mouse major urinary proteins. Protein Sci. 10: 411-417.

North, M. and Pattenden, G. 1990. Synthetic studies towards cyclic peptides: Concise synthesis of thiazoline and thiazole containing amino acids. Tetrahedron 46: 8267-8290.

Novotny, M., Harvey, S., Jemiolo, B., and Alberts, J. 1985. Synthetic pheromones that promote inter-male aggression in mice. Proc. Natl. Acad. Sci. 82: 2059-2061.

Novotny, M., Jemiolo, B., Harvey, S., Wiesler, D., and Marchlewska-Koj, A. 1986. Adrenal-mediated endogenous metabolites inhibit puberty in female mice. Science 231: 722-725.

Novotny, M., Harvey, S., and Jemiolo, B. 1990a. Chemistry of male dominance in the house mouse, Mus domesticus. Experientia 46: 109-113.

Novotny, M., Jemiolo, B., and Harvey, S. 1990b. Chemistry of rodent pheromones: Molecular insights into chemical signaling in mammals. In Chemical signals in vertebrates 5 (ed. D.W. MacDonald, et al.), pp. 1-22. Oxford University Press, Oxford, United Kingdom.

Novotny, M.V., Xie, T.M., Harvey S., Wiesler, D., Jemiolo, B., and Carmack, M. 1995. Stereoselectivity in mammalian chemical communication: Male mouse pheromones. Experientia 51: 738-743.

Novotny, M.V., Ma, W., Jemiolo, B., Wiesler, D., Harvey, S., Xu, F., Xie, T.M., and Carmack, M. 1999a. A unique urinary constituent, 6-hydroxy-6-methyl3-heptanone, has the puberty accelerating pheromone activity in mice. Chem. Biol. 6: 377-383.

Novotny, M.V., Ma, W., Wiesler, D., and Zidek, L. 1999b. Positive identification of the puberty-acceleration pheromone of the house mouse: The volatile ligands associating with the major urinary protein. Proc. Roy. Soc. Lond. B Biol. Sci. 266: 2017-2022.
Payne, C.E., Malone, N., Humphries, R., Bradbrook, C., Veggerby, C., Beynon, R.J., and Hurst, J.L. 2001. Heterogeneity of major urinary proteins in house mice: Population and sex differences. In Chemical signals in vertebrates 9 (eds. A. Marchlewska-Koj et al.), pp. 233-240. Kluwer Academic/Plenum Publishers, New York.

Pelosi, P. 1994. Odorant-binding proteins. Crit. Rev. Biochem. Molec. Biol. 29:199-228.

Pes, D. and Pelosi, P. 1995. Odorant-binding proteins of the mouse. Comp. Biochem. Physiol. B. Biochem. Mol. Biol. 112: 471-479.

Prodromu, C. and Pearl, L.H. 1992. A novel technique for total gene synthesis. Protein Eng. 5: 287-829.

Richieri, G.V., Low P.J., Ogata, R.T., and Kleinfeld, A.M. 1999. Binding kinetics of engineered mutants provide insight about the pathway for entering and exiting the intestinal fatty acid-binding protein. Biochemistry 38: 58885895

Robertson, D., Beynon, R., and Evershed, R. 1993. Extraction, characterization, and binding analysis of two pheromonally active ligands associated with major urinary protein of house mouse (Mus musculus). J. Chem. Ecol. 19: 1405-1416.

Robertson, D., Cox, K.A., Gaskell, S.J., Evershed, R., and Beynon, R. 1996. Molecular heterogeneity in the major urinary proteins of the house mouse Mus musculus. Biochem. J. 316: 265-272.

Ross, J.B.A., Laws, W.R., Rousslang, K.W., and Wyssbrod, H.R. 1992. Tyrosine fluorescence and phosphorescence from proteins and polypeptides. In Topics in fluorescence spectroscopy, biochemical applications (ed. J.R. Lakowicz), pp. 1-63. Plenum Press, New York.

Sampsell, B.M. and Held, W.A. 1985. Variation in the major urinary protein multigene family in wild-derived mice. Genetics 109: 549-568.

Shahan, K., Denaro, M., Gilmartin, M., Shi, Y., and Derman, E. 1987. Expression of six mouse major urinary protein genes in the mammary, parotid, sublingual, submaxillary, and lachrymal glands and in the liver. Mol. Cell Biol. 7: 1947-1954.

Timm, D.E., Baker, L.J., Mueller, H., Zidek, L., and Novotny, M.V. 2001. Structural basis of pheromone binding to mouse major urinary protein (MUP-I). Protein Sci. 10: 997-1004.

Utsumi, M., Ohno, K., Kawasaki, Y., Tamura, M., Kubo, T., and Tohyama,M. 1999. Expression of major urinary protein genes in the nasal glands associated with general olfaction. J. Neurobiol. 39: 227-236.

Wasserman, H.H. and Oku, T. 1986. The carbonyl epoxide rearrangement: A chiral synthesis of the Mus musculus pheromone. Tetrahedron Lett. 27: 4913-4916.

Zidek, L. 1999. "Pheromome-binding proteins in the house mouse." Ph.D. dissertation. Indiana University, Bloomington.

Zidek, L, Stone, M.J., Lato, S.M., Pagel, M.D., Miao, Z., Ellington, A.D., and Novotny, M.V. 1999. NMR mapping of the recombinant mouse major urinary protein I binding site occupied by the pheromone 2-sec-butyl-4,5dihydrothiazole. Biochemistry 38: 9850-9861. 